\title{
The Anatomy of the Knee and Gamma-Families
}

\author{
A.D.Erlykin ${ }^{\mathrm{a}} *$, A.W.Wolfendale ${ }^{\mathrm{b}}$ \\ ${ }^{a}$ P.N.Lebedev Physical Institute Leninsky prosp. 53, Moscow 119991, Russia \\ ${ }^{b}$ Physics Department, University of Durham, South Road, Durham DH1 3LE, UK
}

It is shown that the fine stucture of the cosmic ray energy spectrum in the knee region, if explained by the Single Source Model ( SSM ), can, in principle, be clearly revealed and magnified in the size spectrum of extensive air showers ( EAS ) associated with gamma families. Existing experimental data on EAS at mountain level give support to this hypothesis.

\section{Introduction}

The combination of the original EAS technique with photosensitive materials for the study of the microstructure of EAS cores was proposed long ago [12]3. Since then, in spite of both methods being well developed and widely used in cosmic ray ( CR ) studies they have remained basically independent of each other. However, there have been several attempts to combine them 4/5]6/7/8 9:10/11 and these combinations have brought interesting results. Inspired by these results a few new projects have been proposed to use large stacks of X-ray films within EAS arrays at mountain altitudes, where the intensity and the energy content of both EAS and gammahadron families is much higher than at sea level 121314. In this paper we draw an attention to a promising opportunity offered by this technique.

\section{The Single Source Model of the Knee}

The Single Source Model was proposed by us to explain the fine structure of EAS size spectra in the knee region [15ा16. Besides the sharp knee at EAS sizes $N_{e}$ corresponding to a primary energy of about 3-4 PeV, a second sharp 'peak' ( in $\log \left(I N_{e}^{3}\right)$ vs $\log N_{e}$ coordinates ) has been found at $N_{e}$ corresponding to an energy in the range $12-16 \mathrm{PeV}$. We attribute these structures: the knee and the second 'peak', to a sharp cut-

*E-mail: erlykin@sci.lebedev.ru off of the rigidity spectrum for primary $\mathrm{CR}$ nuclei accelerated by a single, nearby and recent supernova. Initially, we associated the knee with the cutoff of oxygen nuclei and the second 'peak' - with iron nuclei, although we cannot exclude now that they are caused by the energy cutoffs of helium and oxygen [16/17/18. Due to the flat spectrum of the Single Source $(\gamma=2)$ and the sharp cutoffs the peaks in fact look like lines in the EAS size spectra. However, the 'intensities' of these lines are low and they are hardly seen above the background. We look for the possibility of increasing the 'signal to noise ratio' in the ground-based observations using EAS associated with gamma families.

\section{Gamma families initiated by primary nuclei}

The standard attitude is that if one selects EAS above a threshold size $N_{e}^{t h r}$ or a gamma family with a threshold total energy of gamma quanta $\sum_{i}^{n} E_{i}$ then they are induced preferentially by primary protons. It is true, but cannot be extended to shower sizes $N_{e}$ much higher than the threshold $N_{e}^{t h r}$. In Figure 1 we show the probability for primary protons and iron nuclei of different energies creating EAS within a zenith angle interval $\Theta<30^{\circ}$ containing gamma quanta and gamma families at the Tien-Shan altitude ( 3340 $\mathrm{m}$ a.s.l. ). Calculations have been made using the CORSIKA 6.014 package with the QGSGET 
interaction model 19. The multiplicity $n_{\gamma}$ of gamma-quanta in the family has been taken as $n_{\gamma} \geq 1$ and $n_{\gamma} \geq 2$; the minimum energy of gamma-quanta was $4 \mathrm{TeV}$. It is seen that:

(i) in the wide energy region the energy dependence of the probability $P(E)$ is stronger then the direct proportionality $P \propto E$;

(ii) at energies above $10 \mathrm{PeV}$, where the probability for protons to produce the gamma family approaches 1 , the corresponding probability for nuclei also approaches 1 . Therefore, beyond the knee, where according to many experiments the primary CR mass composition is enriched by heavy nuclei, they will be also efficient in producing gamma-families.

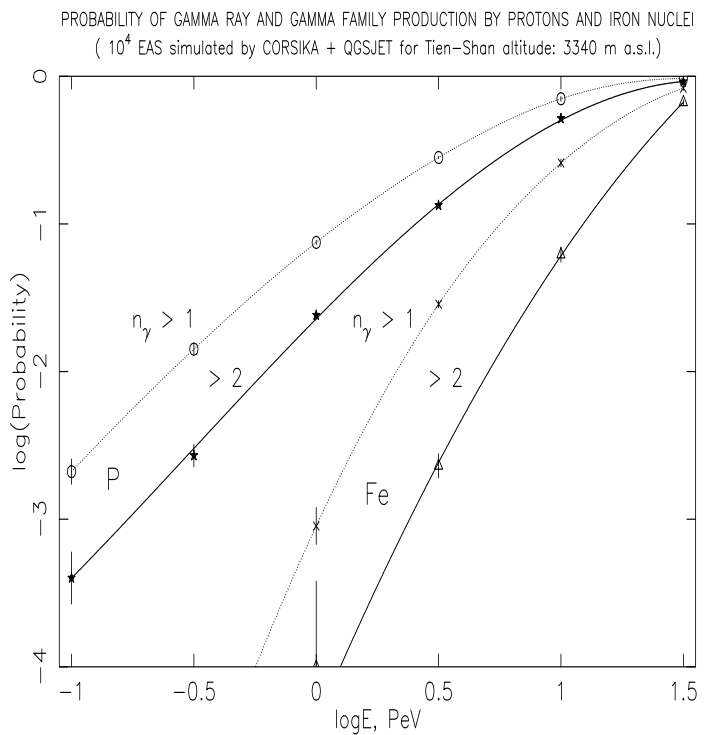

Figure 1. The probability for protons $(\mathrm{P})$ and iron nuclei (Fe) producing gamma-families with multiplicity $n_{\gamma} \geq 1$ and $n_{\gamma} \geq 2$ at Tien-Shan altitude, $3340 \mathrm{~m}$ a.s.l. Open circles: P, $n_{\gamma} \geq 1$, stars - P, $n_{\gamma} \geq 2$, crosses - Fe, $n_{\gamma} \geq 1$, triangles $-\mathrm{Fe}, n_{\gamma} \geq 2$. Dashed and full lines are polynomial fits of the data. The threshold energy of gamma quanta is $4 \mathrm{TeV}$.

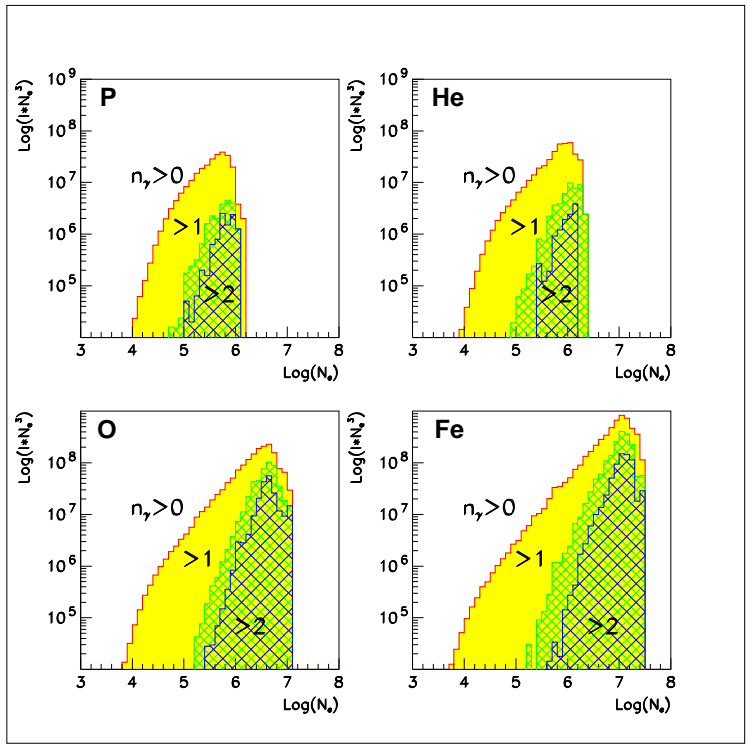

Figure 2. Size spectra of EAS for P, He, O and Fe nuclei (indicated inside the graphs), invoked in the SSM. The histogram with the highest statistics $\left(n_{\gamma} \geq 0\right)$ are for all EAS, histograms with intermediate statistics - EAS containing gamma quanta, i.e. showers with $n_{\gamma} \geq 1$, histograms with lowest statistics - EAS containing gamma families, i.e. showers with $n_{\gamma} \geq 2$.

\section{Size spectra of EAS containing gamma families}

The SSM in common with many other models implies that different CR nuclei are accelerated by a single source up to the same maximum rigidity $R_{\max }$. In particular, in the SSM with helium dominating at the knee $\log R_{\max }, G V=6.2$. In Figure 2 we show the size spectra of EAS produced by protons $\mathrm{P}$, helium $\mathrm{He}$, oxygen $O$ and iron $F e$ nuclei, which can be accelerated by our Single Source. The minimum rigidity $R_{\text {min }}$ has been taken as $0.1 \mathrm{PV}$. The input conditions ( CORSIKA6.014, QGSJET, $\Theta \leq 30^{\circ}$, $\left.E_{\gamma} \geq 4 T e V\right)$ were the same as described in the previous section. Energy spectra within the rigidity intervals have been taken as $\sim E^{-2}$. Showers are shown both containing high energy gamma quanta and families, i.e. for $n_{\gamma} \geq 1,2$, and with- 
out them. It is seen that due to the strong energy dependence of the probability $P(E)$ the size spectra of EAS containing gamma quanta and gamma families are flatter than those for all showers. Since the EAS size cutoff is sharp, these spectra shown in our coordinates $\log \left(I N_{e}^{3}\right)$ vs. $\log N_{e}$ look even more like spectral lines, than the spectra of all showers.

\section{The size spectrum of EAS from the Sin- gle Source}

In Figure 3 we show the spectrum of all showers and showers containing gamma quanta and gamma families for our SSM, i.e. with energy independent abundance $\Delta_{A}$ of different nuclei as $\Delta_{P}=0.004, \Delta_{H e}=0.711, \Delta_{O}=0.210$ and $\Delta_{F e}=0.075$ for fixed rigidity. Due to sharper

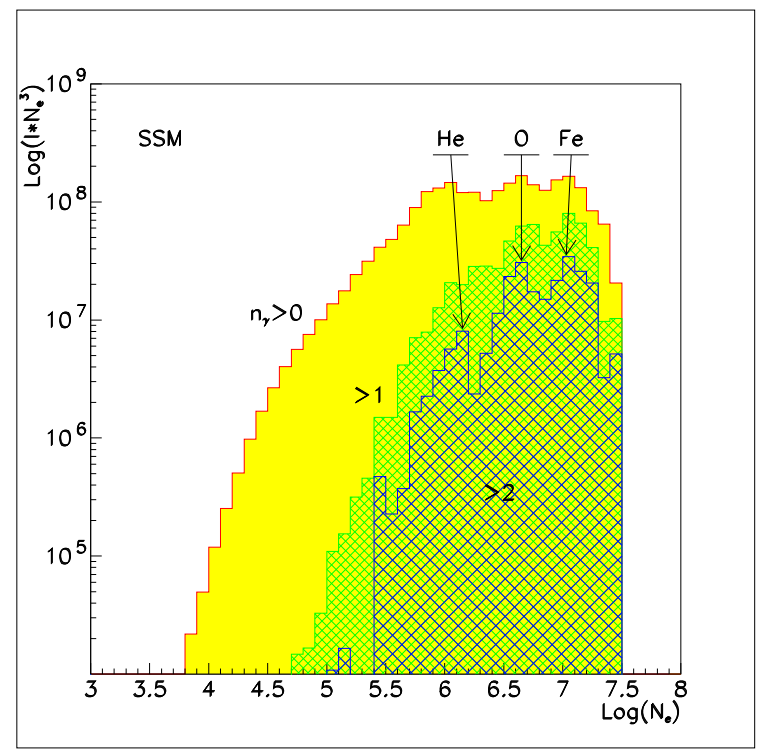

Figure 3. The size spectrum of EAS from the Single Source, which emits $\mathrm{P}, \mathrm{He}, \mathrm{O}$ and Fe nuclei with constant abundances: 0.004 for $\mathrm{P}, 0.711$ for $\mathrm{He}, 0.210$ for $\mathrm{O}$ and 0.075 for Fe. The relevant histograms for EAS are the same as in Figure 2. The location of the peaks is indicated.

peaks in the individual spectra related to the en- ergy cutoffs of different nuclei in CR from the Single Source these peaks are more distinct in the spectrum of EAS containing gamma families rather than in the total spectrum of all EAS. As can be seen in Figure 3 the excess over the smooth background in the spectrum of all showers in the logarithmic scale is about $\Delta\left(\log I N_{e}^{3}\right)=$ $0.18 \pm 0.01$ ( signal/noise ratio $S / N \simeq 0.5$ ), while that in the spectrum of showers containing gamma families is about $0.36 \pm 0.02(S / N \simeq 1.3)$. Of course, the statistics of the showers with families is less than that of all showers and, besides the smooth background of the Single Source, there is a background from other sources, which reduces the $S / N$ ratio. However, the estimates based on our simulations show that the confidence level of detecting the signal in the size spectrum of EAS with families is about the same as in the spectrum of all EAS.

There is experimental evidence that in the knee region the size spectrum of EAS with gamma families observed at mountain altitude has sharp peaks, which are more clearly visible than irregularities in the spectrum of all showers [20. Since this Tien-Shan experiment has the highest statistics of such showers in the world, this result deserves further analysis and the technique of the combined use of EAS detectors with X-ray films - a further development and longer exposure.

Reliable distinction of proton- and helium- induced peaks can help to make a choice between models of the cosmic ray origin in which cut-off energies in the spectra of constituent nuclei are proportional to their charges [21] or masses [22].

\section{Conclusion}

It is shown that if there is a sharp cutoff in the rigidity spectrum of accelerated nuclei, as in the Single Source Model of the knee, then the size spectrum of the EAS containing gamma families can have more distinct peaks corresponding to the cutoffs of different nuclei than the spectrum of all EAS. There is experimental evidence for the existence of such peaks and the present paper is to some extent inspired by this unique observation. 


\section{REFERENCES}

1. Brikker S.I. et al., 1958, Suppl. Nuovo Cim., 8, 733

2. Rappoport I.D., 1958, J. Exp. Theor. Phys., 34, 998

3. Grigorov N.L. et al., 1959, 6th Int. Cosm. Ray. Conf., Moscow, 1, 122

4. Smorodin Yu.A. et al., 1965, 9th Int. Cosm. Ray Conf., London, 2, 827

5. Matano T. et al., 1970, Acta Phys. Hung., 29, 451

6. Dake S. et al., 1970, Acta Phys. Hung., 29, 671

7. Dake S. et al., 1977, Nuovo Cim., 41, 55

8. Ohta K. et al., 1979, 16th Int. Cosm. Ray Conf., Kyoto, 13, 177

9. Shaulov S.B. et al., 1986, Vopr. Atom. Nauk. Techn., ser. Techn. Phys. Exp., 3(29), 72

10. Adamov D.S. et al., 1987, 20th Int. Cosm. Ray Conf., Moscow, 6, 144

11. Kawasumi N. et al., 1996, Phys. Rev. D, 53, 3534

12. Aoki H. et al., 2005, Nucl. Phys. B ( Proc. Suppl. ) ( to be published )

13. Saavedra O. and Jones L.W., 2001, Nuovo Cim. C24, 497

14. Amurina A. et al., 2005, Nucl. Phys. B. ( Proc. Suppl. ) ( to be published )

15. Erlykin A.D. and Wolfendale A.W., 1997, J. Phys. G: Nucl. Part.Phys., 23, 979

16. Erlykin A.D. and Wolfendale A.W., 2001, J. Phys. G: Nucl. Part.Phys., 27, 1005

17. Erlykin A.D. and Wolfendale A.W., 2005, Astropart. Phys., 23, 1

18. Erlykin A.D. and Wolfendale A.W., 2005, submitted to Phys. Lett. B

19. Heck D. and Knapp J., EAS Simulation with CORSIKA: A User Guide ( version 6.014 from 25.02.2002 ), FZK

20. Shaulov S.B., 2001, 27th Int. Cosm. Ray Conf., Hamburg, 1, 116

21. Vólk H.J., 2001, astro-ph/00105355

22. Dar A. et al. 2006, Nucl. Phys. B. ( Proc. Suppl. ), 2007 ( to be published ), astro-ph/0606199 